\title{
Design of a passive RFID based Sensor System
}

\author{
Simon Harasty ${ }^{1}$, Christian Arnold ${ }^{2}$, Bolli Björnsson ${ }^{3}$ \\ ${ }^{1}$ University of applied Science Fulda, Marquardstraße 35, 36037 Fulda, Germany, \\ Simon.Harasty@et.hs-fulda.de \\ ${ }^{2}$ University of applied Science Fulda, Marquardstraße 35, 36037 Fulda, Germany, \\ Christian.Arnold@et.hs-fulda.de \\ ${ }^{3}$ University of applied Science Fulda, Marquardstraße 35, 36037 Fulda, Germany, \\ Bolli.Bjoernsson@et.hs-fulda.de
}

\begin{abstract}
RFID (Radio Frequency Identification) is a common technology for wireless object identifying. It is used in many industrial automation applications, like in stock management and distribution. The simple concept of an RFID system is the usage of a coupled magnetic field to generate the power supply for the transponder which is attached to the object. Hereby the integration of a sensor to a transponder appears to be a good possibility to rise near object measurements with a passive energy supply. A simple conception of passive RFID based sensor system will be shown in this contribution.
\end{abstract}

Key words: passive sensor, RFID, HF

\section{Introduction}

RFID is a wireless Auto-ID-System for identifying objects like barcodes or magnetic strip solutions. The advantage of RFID over the classic identifying system is, that there is no visual or direct contact needed to identify an object and group detection for multiple RFID devices is possible. For secured applications there are different ciphering methods available to grant secured access.

By now the RFID-technology is common in many industrial automation applications, like in stock management, distribution and access control of secured areas. In all applications are more efficient RFID solutions offered to optimize logistic processes (see [1]).

The focus of current researches and developments is on the combination of RFID and sensor technology. First solutions are already presented in medical applications. The usage of this system in automation processes is moderate, founded to the high costs of special designed products, which results in a high development potential. In this contribution the possibilities and advantages of passive RFID sensor systems are pointed out.

Especially in automation technology, the application of such systems has a high potential for optimization of manufacturing processes. It can be used to identify product states and determine next production steps. In these production processes no connection to databases is needed, because the product itself contains instructions for future production steps. Furthermore, new options and applications arise from the possibility of local data collection of prepackaged products or by capturing product states from separated areas (see [2]).

\section{System types}

The main parts of an RFID system are the RFID-reader and the transponders (tag). The reader provides a magnetic field, which is used for the communication and energy assigning. There are three system types which are differenced by the tags energy supply. Using an additional power source, such as a battery to supply the tags logic units and to support the tags wireless communication, the system is called an active system. An active system has the highest communication range due to its own power source, but they are also the largest and most expensive system types (see [3]).

A system using only the energy of the reader's magnetic field as well for the communication as for supplying the tags electronics is called passive. Simple passive tags for identifying can be very small and produced with low costs. Due to the lag of a supported communication its operating range is smaller than an active system. Also an energy efficient tag design is recommended because the field energy is limited by country regulations (see [1] and [3]).

Is an additional power source only used to support logic units or additional electronic parts, 
but not for supporting the communication, the system is called semi-active or semi-passive. In a semi-passive system the energy needed for the communication is obtained to the tag by the reader's magnetic field (see [1]).

Another characteristic in RFID systems is the system frequency on which the systems operating range, the size of the parts and the price depends.

Low frequency systems (LF) operate in a frequency range from $30 \mathrm{up} \mathrm{to} 300 \mathrm{kHz}$. Typical frequencies for RFID systems are 125 or 135 $\mathrm{kHz}$. Low frequency systems have a good controllability contingent on the little parasitic effects on lower frequencies. Due to the needed high inductance of the antenna LF system tags are large compared to the other types (see [1]).

High frequency systems (HF), like in the presented System, use a typical frequency of 13.56 MHz. Due to the higher frequency there are more parasitic effects to consider by designing a system. LF and HF systems are inductive coupled like a transformer (see Essentials), which limits the operating range to the readers magnetic field. The size of the magnetic field depends on the frequency's wavelength (see [1]).

Systems with higher frequencies are ultra-highfrequency (UHF) and microwave (MW) systems, which are coupled electromagnetic. These types of systems have the highest communication ranges, but also the highest production and development costs.

The benefit of passive RFID sensor systems is the possibility of a completely wireless measurement. It is possible to measure system states in separate areas as behind walls. The data may be captured without a local power source. This contrasts with the disadvantage that for measuring a reader's magnetic field is needed to supply the transponder. So the system is not useful for condition monitoring by the lag of the possibility of a constant measurement. Applications for passive sensors may be found in production processes where internal state data during production is needed (see [2]).

Special attention must be paid to the energy budget of the transponder. The transponder used sensor must be chosen as energy efficient as possible to minimize the needed energy for operating, to increase the operating range of the system.

To get a passive monitoring system, disposable transponders were developed. There are tags for monitoring certain limits of temperature, humidity or acceleration. Materials are used, which change their characteristics with the occurring physical sizes. After exceeding a limit value the material changes its electric characteristic in such a way that it can be detected by special readers. Thereby, exceeding a value limit during storage or shipment can be detected. The costs for such transponders are comparatively high, so its usage seems only reasonable for goods with a high worth. Beside the higher costs the measurands of such a system are severely limited.

\section{Essentials}

HF and LF systems are coupled inductive like in a transformer. The Reader provides a magnetic field of the systems frequency. This field accrues at the reader antenna and is coupled with systems within the field range as shown in figure 1.

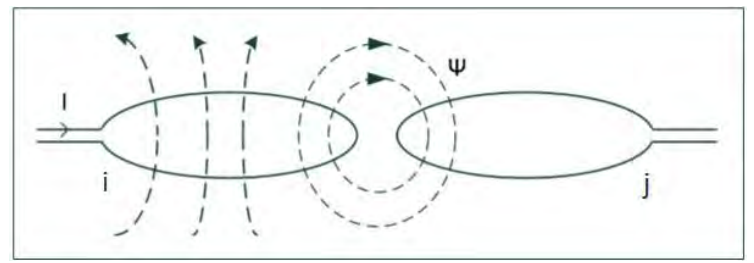

Fig. 1 Mutual inductance

The strength of the magnetic field is defined by the magnetic flux $\Psi$ as a function of the current $I$ in the reader antenna coil. The induced voltage to the tags antenna coil can be determined by equation 1 .

$u_{i}=\frac{\partial \psi(t)}{\partial t}$

The range of the magnetic field is limited by the wavelength $\lambda$ of the system as function of the frequency. At a distance $d$ as shown in equation 2 the magnetic field skips to an electromagnetic field, so that an inductive coupling is no longer possible (see [1]).

$d>\frac{\lambda}{2 \cdot \pi}$

The assigned power of the system is influenced by the Antennas Q-factor, depending on the antenna resistance (see equation 3 ), where a higher Q-factor indicates a better power consumption.

$Q=\frac{R_{L}}{2 \cdot \pi \cdot f_{0} \cdot L}$

The provided power on the tag can be seen by the magnetic field strength $\mathrm{H}$. At a simplified coil system model like shown in figure 1 the 
strength of the magnetic field depends on the distance $d$ of the coils as shown in figure 2 .

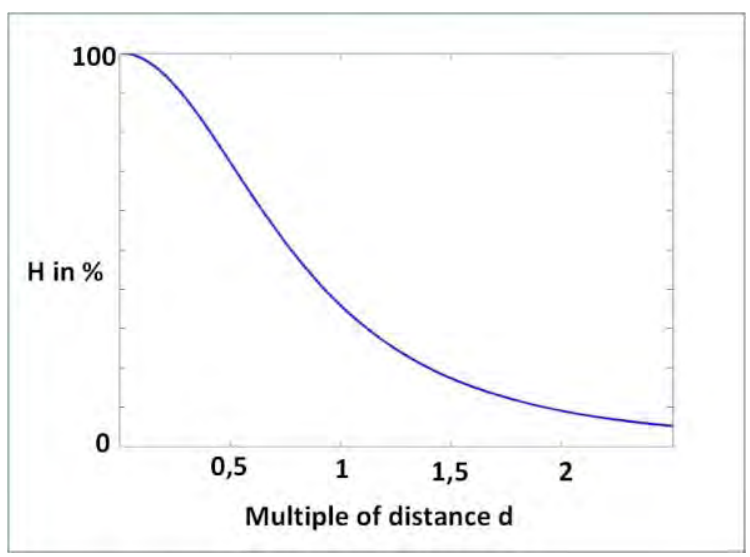

Fig. 2 Magnetic field strength

\section{Antenna design}

An important system characteristic is the communication range of the System. The biggest factor beside the signal strength for the range is given by the size of the reader and transponder antennas. The choices of the antenna parameters are influencing the whole system design by the antenna adjustment circuit. Typically rectangular antennas are used for HF RFID systems, because of the design which can be realized on printed circuit boards. For designing a rectangular antenna and determining its main properties like inductance and resistance there are useful application notes by different corporations like [4] and [5].

To fit the antenna circuit to the used frequency for a first approach equation 3 can be used. For a more exact fitting the usage of a LCR meter is recommended to optimize the behavior of the oscillating circuit for a specific bandwidth.

$f_{0}=\frac{1}{2 \cdot \pi \cdot \sqrt{L \cdot C}}$

To determine the range of an RFID system the ratio of the reader and transponder antenna has to be considered. The ratio considers the area and number of turns of the rectangular antennas. For testing the influence of the ratio a measurement of different transponder antennas was done with two reader antennas by a transmission power of $200 \mathrm{~mW}$. As to see in figure 3 a lower ratio gives a better system range. So that more similar antennas can lead to a better range.

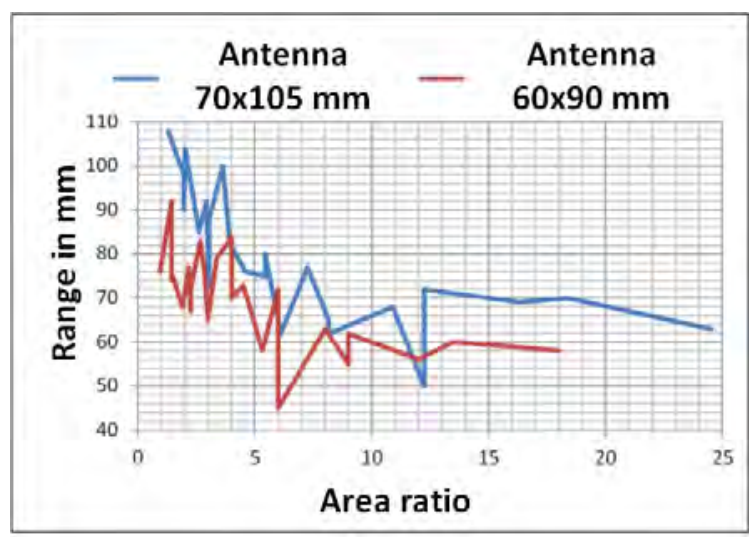

Fig. 3 Magnetic field strength

\section{Communication}

The communication in RFID Systems is standardized for the different fields of applications. The most common use are the ISO 14443 (contactless systems, see [6]), ISO 15693 (vincinity cards, see [7]) and ISO 18000 (physical transmission, see [8]). There is no standard explicit for sensor systems, so that in the here presented systems an own developed data structure is used, which is based on this standards.

The communication between reader and tag is realized by amplitude modulation (Amplitude Shift Keying; ASK). So, the data is modulated by lowering the magnetic fields amplitude. The transmission from tag to reader is realized by a $10 \%$ ASK, which means the amplitude is lowered by $10 \%$ for a signal. The data send from the reader to the transponder is modulated with a $100 \%$ ASK or OOK (On-Off-Keying), so that there is no amplitude when a signal is modulated.

\section{Tag Design}

The RFID transponder presented in this contribution is based on a standard microcontroller. Especially the rectification, modulation and the power handling of the transponder are important for a simple tag design.

Reader and transponder system components and their development effort should be minimized by using standardized methods of communication such as ISO 15693 [7], this allows existing reader and transponder chips to be used. This section describes a simple passive system without special transponder chip to provide a more precise definition of the interface aligned for sensor usage.

To get a simple realization (see figure 4) only one pin of the microcontroller is used for modulation demodulation and supplying the transponder with the needed power. The used 
antenna is aligned with capacitor C1 to 13.56 $\mathrm{MHz}$, whereby voltage is induced while the transponder is in a reader's field. The signal is rectified by diode $\mathrm{D} 1$ and abraded by a low pass filter consisting of R1, C2 and R2. The resistor R1 is also used to adjust the modulation intensity. Over the controller intern clamping diode D2 the used pin is coupled with the supply voltage. The microcontroller is decoupled from other parts in the circuit over capacitor C3.

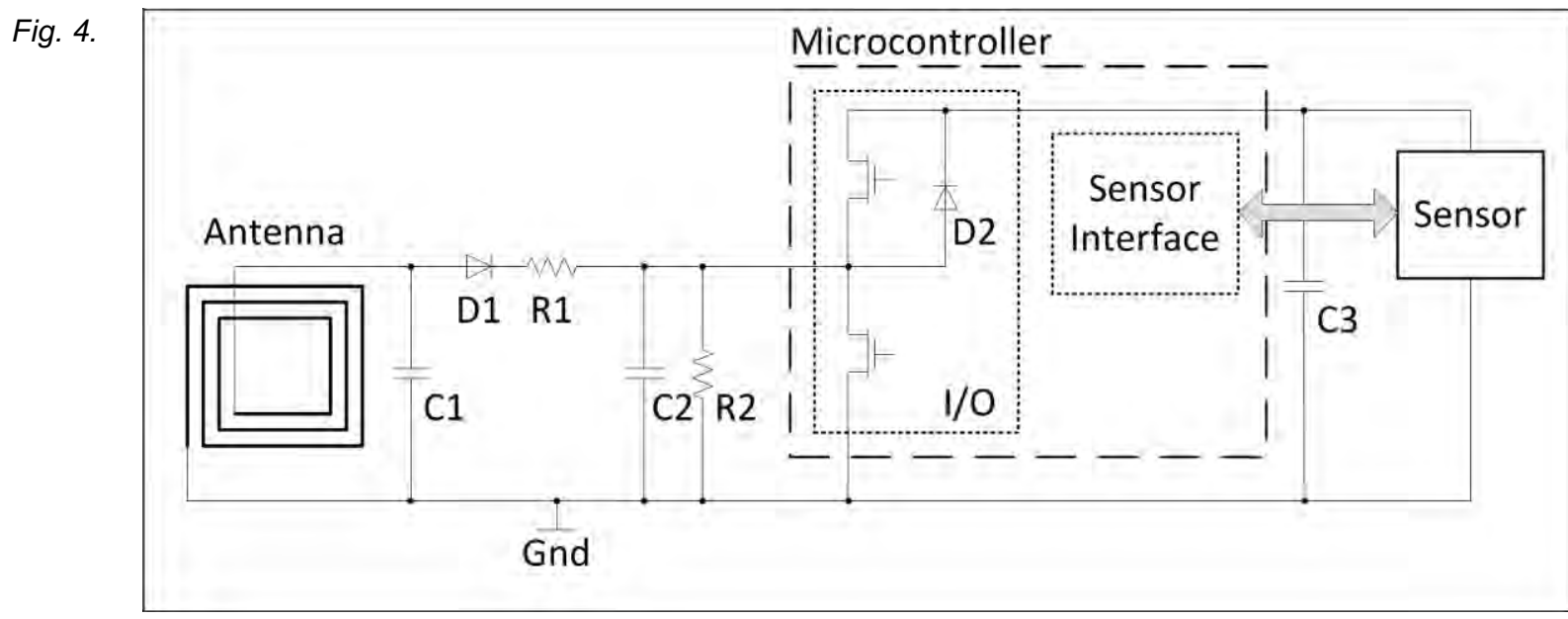

Circuit for passive RFID Sensor

To modulate the field, the used pin is dragged to low, generating a higher load on the antenna. A modulated OOK signal can simply be detect as a low signal on the used pin.

Now the generated power at the transponder can be used to attach sensors to the microcontroller. Most controllers provide some $A / D$ converters, which are quite useful for getting sensor data. Either, connecting a sensor and using the $A / D$ converter need a power handling for storing energy and limit voltage. These also simply can be provided by an extra capacitor and a zener diode. For exact measurements depending on voltage levels a further stabilization for the voltage is needed.

In the system in the here provided paper the usage of a software $\mathrm{I}^{2} \mathrm{C}$-bus for a temperature and humidity sensor was used (see figure 6).

The main usage for such a passive supplied sensor is the measuring of inner object states. For Example it allows measuring the humidity inside of a wall over a long time period without the maintenance for service.

\section{Reader design}

The construction of a reader is done with a Standard RFID reader chip to the system requirements, such as transmission power should be selected. The purpose of this is to provide chip reader of the magnetic field from an antenna as well as the detection of modulated signals. The demodulated signals are interpreted by a microcontroller and for example transmitted to a computer.
The reader used is designed as an USB stick to use it directly on a computer. The reader stick has a virtual serial COM-port as an USB interface. The used microcontroller can so be used with a simple USART (Universal Synchronous/Asynchronous Receiver Transceiver), which is present in most microcontrollers.

The here used Stick (see figure 5 and figure 6) uses an ATMega644 microcontroller $(\mu \mathrm{C})$, which provides the interface for an analog front end chip by EM Microelectronics. The front end chip establishes the field for the antenna, modulates transmission for the tag and demodulates transmissions from the tag. The interface between the microcontroller and the front end chip is realized by an SPI bus (Serial peripheral interface).

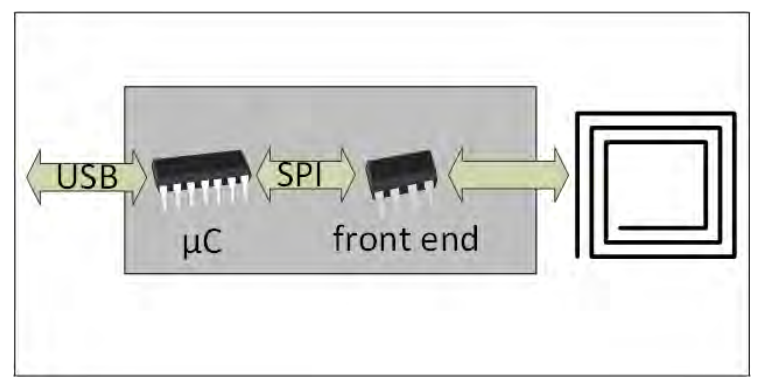

Fig. 5 Structure for RFID reader

The data received from the tag is calculated to the physical value of the measurement and transferred as string via the virtual serial interface to the computer. By doing so no special software is needed to use the system 
and it may be included easily in existing systems.

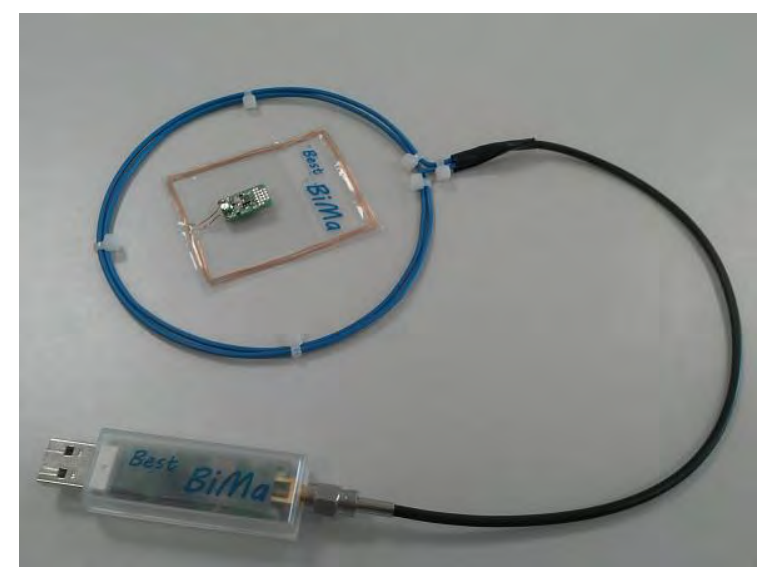

Fig. 6 RFID Reader and Tag

\section{Conclusion}

In the here presented paper the design of a simple RFID sensor system is shown. This type of system allows measurements without an additional power source on the transponder. This type of wireless measurement can be used as platform for several sensors with low power consumption.

The building of a complete system is a complex task, which needs special skills in different areas of electronic design, but by the usage of simplified rules, like shown here, the development of a prototype like system may be done. To develop a complete system a high effort in designing the antennas and its fitting circuit is needed, but the usage of a microcontroller as controlling unit is very valuable. By using its inner circuits for supporting the rectifying, modulating and demodulating a one wire RFID Interface is realized.

The usage of a simple microcontroller allows the system to be adapted to the needs of the application. By fitting the tags voltage for a sensor system it can be used for standard measurements like resistance measurements. For capacitive measurements a stable voltage is essential to determine a precise value.

To do measurements with a higher need of energy the accumulation of energy over a time period seems to be an successful option. Using a capacitor with a capacity high enough for the determined measurement may be loaded over a time period and the stored energy may be used for a measurement.

\section{References}

[1] Klaus Finkenzeller: RFID Handbook: Fundamentals and Applications in Contactless Smart Cards and Identification, Third Edition. John Wiley and sons 2010

[2] Harasty, S., Arnold C., Björnsson, B., Michelmann, N.: Entwicklung von passiven und semi-passiven RFID-Sensorsystemen für die Automatisierungstechnik, Automation 2011

[3] Weinstein, Ron: RFID: S Technical Overview and Its Application to the Enterprise, IT Pro, IEEE Computer Society, 2005

[4] Melexis: Antenna Design for MLX90129, Rev 01, Melexis, 2010, AN 390119012903

[5] Lee Youbok: Antenna Circuit Design for RFID Applications, Microchip, 2003, AN710

[6] International Standardization Organisation: ISO/IEC 14443 Identification cards Contactless integrated circuit cards Proximity cards, 2008

[7] International Standardization Organisation: ISO/IEC 15693 Identification cards Contactless integrated circuit cardsvincinity cards, 2010

[8] International Standardization Organisation: ISO/IEC 18000 Radio frequency identification for item management, 2008 